\title{
Selection on olfactory response to semiochemicals from a plant-host complex in a parasitic wasp
}

\author{
Q Wang, H Gu and S Dorn \\ Institute of Plant Sciences/Applied Entomology, Swiss Federal Institute of Technology (ETH), Zurich, Switzerland
}

Parasitic wasps (parasitoids) use volatiles from the plants infested by phytophagous insects to locate host herbivores, but their behavioural response to such semiochemicals is highly variable. Bi-directional selection on Cotesia glomerata (Hymenoptera: Braconidae) was conducted to investigate the importance of genetic variation in the olfactory response of parasitoids. Female wasps were assessed for flight orientation and landing success in response to the hexane extract from a plant-host complex in a wind tunnel. After the first generation of selection, two strains significantly differentiated in both flight orientation and landing success, and their divergence continued with further selection. The two selected strains genetically differentiated in olfactory perception rather than upwind flight ability. The realized heritability was estimated as 0.248 for flight orientation and 0.216 for landing success. The selection experiment further demonstrated that a prior exposure to the semiochemicals significantly enhanced the subsequent response of female wasps, independent of genetic differences. These results suggest that both genetic component and environmental conditioning have played an important role in the evolution of host selection and utilization by the parasitoid in a tritrophic system.

Heredity (2003) 91, 430-435. doi:10.1038/sj.hdy.6800340

Keywords: parasitoid; Cotesia glomerata; olfactory response; semiochemicals; selection; realized heritability

\section{Introduction}

Parasitic wasps (parasitoids) have evolved the ability to use volatiles from herbivore-infested plants in the process of host selection (Vinson, 1976; Turlings et al, 1990; Dicke, 1994). Based on quantitative and/or qualitative differences in the volatile composition, parasitoids can even distinguish the plants infested by their hosts from those infested by closely related nonhost species (De Moraes et al, 1998). On the other hand, the behavioural response of parasitoids to such semiochemicals can be highly variable among individuals of a single species due to differences in genotype, physiological status and experience (Lewis et al, 1990). Intraspecific variation in the odour-guided behaviour of parasitoids is presumably adaptive, especially for the generalist species, which have multiple, polyphagous hosts, in the context of their habitat characteristics (Vet and Dicke, 1992). To understand the ecological and evolutionary significance of such variation, a genetic analysis is essential, as it is the genetic variance that provides the raw material for any response to natural selection (Falconer, 1989).

The analysis of isofemale strains and the comparison of geographic strains have demonstrated genetic variation in the olfactory response of parasitoids. By comparing four isofemale strains for three generations, Prevost and Lewis (1990) found that flight response to volatiles from a plant-host complex was genetically controlled in

Correspondence: H Gu, Institute of Plant Sciences/Applied Entomology, Swiss Federal Institute of Technology (ETH), Clausiusstrasse 25, NW, CH-8092 Zurich, Switzerland. E-mail: hainan.gu@ipw.agrl.ethz.ch

Received 25 March 2003 the parasitic wasp Microplitis croceipes (Cresson). However, this work relied on the response of experienced individuals, that is, wasps with pre-exposure to leaves of the host-infested cowpea seedling contaminated with host faeces. Gu and Dorn (2000) conducted a similar analysis of isofemale strains established from a field population in the parasitic wasp Cotesia glomerata (L.), in which they examined the flight response of naïve female wasps to a host-infested plant, that is, the wasps had no contact with any host and host-plant cues prior to bioassay. This study revealed the presence of an additive genetic variance in the innate olfactory response of the parasitoid. Very recently, Campan et al (2002) compared two geographic strains (one from Nasrallah, Tunisia, and the other from Brazzaville, Congo) of the parasitic wasp Leptopilina boulardi Barbotin et al and their hybrids for the innate ovipositor probing behaviour in response to fruit aromas. The results of this study showed that the probability of probing was subject to an apparent complete dominance of Nasrallah characteristics and additive inheritance in the Brazzaville lineage. Furthermore, these sporadic studies have provided empirical evidence that olfactory response in parasitoids is largely a quantitative character.

The present study represents the first attempt to apply the approach of experimental selection to investigation into the role of genetic variation in the olfactory response of parasitoids. The parasitic wasp C. glomerata was used as a model species, as the olfactory response to semiochemicals from cabbage plants infested from the host herbivores has been well documented. A Brassica plant attacked by Pieris caterpillars emits a blend of volatiles that is different from the blend emitted by undamaged plants, and volatiles from the plant-host 
complex attract the female wasps (Mattiacci et al, 1995, 2001). In wind-tunnel tests, female C. glomerata wasps display oriented flights to the volatile chemicals emitted from cabbage plants (Brassica oleracea) infested by the cabbage butterfly (Pieris brassicae) (Steinberg et al, 1992; $\mathrm{Gu}$ and Dorn, 2001). Based on a standardized bioassay in a wind tunnel, bidirectional selection was carried out for the response of the female wasps to the hexane extract from a plant-host complex. The selection experiment was also designed to examine the effect of pre-exposure to the semiochemicals on the subsequent response of wasps in relation to their genetic differences. An additional experiment was conducted to compare the behavioural response of the two selected strains to the solvent alone versus the hexane extract. This experiment was designed to test the hypothesis that selection was not imposed on upwind flight ability and that the observed differences in flight response resulted from differentiation in olfactory perception.

\section{Materials and methods}

\section{Experimental insects}

Cotesia glomerata is a gregarious larval endoparasitoid of several Pieris spp worldwide (Laing and Levin, 1982). The parasitoid samples were collected from a field population parasitizing $P$. brassicae in Canton Zurich, Switzerland. The host herbivore $P$. brassicae was obtained from a laboratory colony and reared on cabbage leaves. After one generation of rearing in the insectary at $21 \pm 1^{\circ} \mathrm{C}, 40-60 \%$ relative humidity (r.h.) and L16:D8, the parasitoid colony was induced into diapause and maintained at $5 \pm 1^{\circ} \mathrm{C}$ in a cold chamber for 5 months prior to use in the selection experiment.

For the experiment, three to five cocoons were taken from each of 150-200 diapausing parasitoid broods, mixed and transferred into an environmental chamber at $21^{\circ} \mathrm{C}, 70 \%$ r.h. and L16:D8 for emergence. Newly emerged males and females were fed with water and honey in nylon gauze cages $\left(30 \times 30 \times 30 \mathrm{~cm}^{3}\right)$. After 5 days, mated female wasps were randomly selected and transferred to plastic boxes $\left(20 \times 10 \times 8.5 \mathrm{~cm}^{3}\right)$, with one female per box. Mesh windows were available on the top or sides of each box for ventilation. Each female was offered five second instar P. brassicae for parasitism. As such, 40 full-sib families were established. Adult wasps were deprived of any contact with plant- or host-related cues before bioassay.

\section{Wind-tunnel bioassay}

The olfactory response of parasitoid wasps was assessed in a wind tunnel $\left(150 \times 35 \times 35 \mathrm{~cm}^{3}\right)$. To standardize the bioassay, the hexane extract from a plant-host complex was used as an odour source. The plant-host complex consisted of host faeces, host exuviae and host-infested cabbage leaves. Host faeces and exuviae were obtained from the second instar $P$. brassicae caterpillars feeding on cabbage plants (Brassica oleracea var. capitata cv. Mezzo), which had grown in a greenhouse for 6-7 weeks. Cabbage leaves were taken from these host-infested plants. The plant-host complex, containing $5.2 \mathrm{~g}$ host faeces, $0.16 \mathrm{~g}$ host exuviae and $1.2 \mathrm{~g}$ cabbage leaves, was soaked in $108 \mathrm{ml} 99.5 \%$ hexane at room temperature for $30 \mathrm{~min}$ and then left at $-60^{\circ} \mathrm{C}$ for $36 \mathrm{~h}$. The supernatant was stored in $0.5 \mathrm{ml}$ aliquots in $2 \mathrm{ml}$ glass vials (clear screw top vial with a $10-\mathrm{mm}$ opening, red PTFE/silicone septa, from SUPELCO) at $-60^{\circ} \mathrm{C}$ for use throughout this study.

A volatile-releasing system was developed for the wind-tunnel bioassay. A small strip of Whatman filter paper was inserted as a wick into a $100-\mu 1$ micropipette, with the filter paper wick extending beyond the two open ends. A small hole was drilled in the septum of a 2-ml glass vial containing the hexane extract and the micropipette with the wick was inserted so that the wick came into direct contact with the extract. The vial was positioned at about $45^{\circ}$ relative to the windtunnel floor upwind, and wasps were released individually downwind on a platform $50 \mathrm{~cm}$ from the odour source. The release platform was adjusted to the same height as the odour source. To exclude visual cues, a white gauze curtain was set up in front of the odour source.

Virgin female wasps (4-day old) were tested for flight response at a temperature of $20 \pm 1{ }^{\circ} \mathrm{C}$, r.h of $50-60 \%$, wind speed of $30-35 \mathrm{~cm} / \mathrm{s}$ and light intensity at 640 650 lux (Gu and Dorn, 2001). A total of 50-145 wasps were tested individually for each strain in each generation. Each individual was given a chance to take off from the release platform. After take-off, the wasp was observed until it landed on the gauze or elsewhere. An observation was terminated if the wasp did not take off within a maximum of $2 \mathrm{~min}$ after release, and this was scored as no response. A straightforward or zigzagging flight towards the odour source was recorded as 'flight orientation' (Gu and Dorn, 2000). If the oriented flight led to a final landing on the gauze, it was recorded as 'landing success'. A random flying performance was considered failure in flight response to the odour source. The first observation measured the innate response of the wasps as they were naïve. In most previous studies on olfactory response in parasitoids, however, behavioural tests who normally conducted on the individuals who had had an exposure to a plant-host complex and even an experience of oviposition on their hosts (eg Steinberg et al, 1992). In order to know how a prior exposure to volatiles from a plant-host complex affected the innate response of naïve wasps in different genotypes, following the first observation, each wasp was offered a second chance to respond to the odour source. This was referred to as the second observation.

\section{Selection procedure}

A family selection procedure was applied, that is, a whole family was selected or rejected as a unit according to its mean phenotypic value (Falconer, 1989). The selection experiment was initiated with the 40 full-sib families derived from the diapaused cocoon clusters (see above). Among these families, 10 families showing the highest level of response, measured in the first observation, were selected for the upward direction, 10 families showing the lowest level of response were selected for the downward direction, and other families were discarded. From each of these 10 families, four female wasps were selected as mothers to mate and reproduce; therefore, 40 families were set up again for each selected strain (ie direction) in the F1 generation. In this way, each following generation was always started with 40 families for each selected strain. 
In order to prevent sibmating, individual cocoons were separated from each cluster before emergence. Usually, up to 30 cocoons were taken from each family. Cocoons were singly placed in plastic tubes $(6 \mathrm{ml})$ to obtain virgin wasps for flight test. The tested female virgins from each selected family were allowed to mate only with males from other families showing a corresponding level of flight response within the same strain. After being kept together with males for 2 days, each mated female wasp was transferred into a plastic box $\left(20 \times 10 \times 8.5 \mathrm{~cm}^{3}\right)$ and offered five second instar caterpillars for parasitism. This procedure not only minimized inbreeding but also allowed consideration of a possible paternal effect on progeny performance, even though only females were testable for the character concerned.

Test for flight response to hexane extract versus hexane alone in the two selected strains

It was hypothesized that the significantly diverged flight orientation and landing success of the two selected strains resulted from their differences in olfactory response rather than upwind flight ability. In order to test this hypothesis, behavioural responses to the hexane extract and to a $99.5 \%$ hexane solvent alone (control) were evaluated in the wind tunnel, respectively. The bioassay condition was the same as previously described for the bidirectional selection, with the hexane extract or the solvent alone placed upwind $50 \mathrm{~cm}$ away from the release platform. Virgin female wasps (4-day old) fed on honey and water were used in the bioassay as well. In total, 31-40 females from each strain were tested individually for their response either to the hexane extract or to the solvent alone. Behavioural responses were measured in terms of flight orientation and landing success, and scored according to the criteria defined for the bidirectional selection. However, each wasp was tested once in this case, so that only the innate behavioural response was taken into consideration. Behavioural responses to the hexane extract and to the solvent were compared within each strain and also evaluated between the two strains.

\section{Statistical analysis}

Data analyses were conducted using the statistical program StatView ${ }^{\mathbb{B}}$ unless otherwise stated. The ANOVA with repeated measures (ie generations) was performed to discern differentiation between the selected strains and differences between responses recorded in the first and second observations. A $t$-test was used to compare differences between the high- and low-olfactory response strains after the first generation of selection. All data were subjected to arcsine transformation prior to analysis. Based on the results of the first observation, the realized heritability was estimated for the innate olfactory response by calculating the ratio $R / S$ ( $R$-the response to the upward or downward selection, $S$-the selection differential) (Falconer, 1989). Logistic regression of SPSS 10.0 for Macintosh was applied to the binary data to analyse differences in behavioural response to the hexane extract and to the hexane solvent within each strain and to evaluate differences between the two strains.

\section{Results}

Changes in innate flight response due to selection The first observation measured the innate flight response of parasitoids to the hexane extract. Directional selection for high- and low-olfactory responses resulted in the divergence of two C. glomerata strains in flight orientation and landing success (Figure 1). After the first generation (F1) of selection, the two strains already differed in the level of both flight orientation $(t=5.17, \mathrm{df}=28, P<0.001)$ and landing success $(t=2.96, \mathrm{df}=28, P<0.01)$. Their divergence continued as further selection was imposed. After four generations of selection, the level of flight orientation differed significantly between strains $(\mathrm{F}=53.04, \mathrm{df}=1,19, P<0.001)$ and between generations $(\mathrm{F}=3.17, \mathrm{df}=4,76, \quad P<0.05)$, although there were interactions between strain and generation $(\mathrm{F}=3.25$, $\mathrm{df}=4,76, P<0.05)$.

Not all individuals displaying flight orientation to the odour source successfully landed on the gauze, and thus percentages of landing wasps were lower than those of wasps showing flight orientation in each generation. However, generational changes in landing success due to selection showed a pattern similar to that in flight orientation (Figure 1), and the two strains also significantly differed in landing success after four generations of selection $(\mathrm{F}=20.53 \mathrm{df}=1,19, P<0.001)$.

The heritability estimated for flight orientation was much higher from the response to upward selection than from the response to downward selection, but an opposite trend was shown for landing success (Table 1).
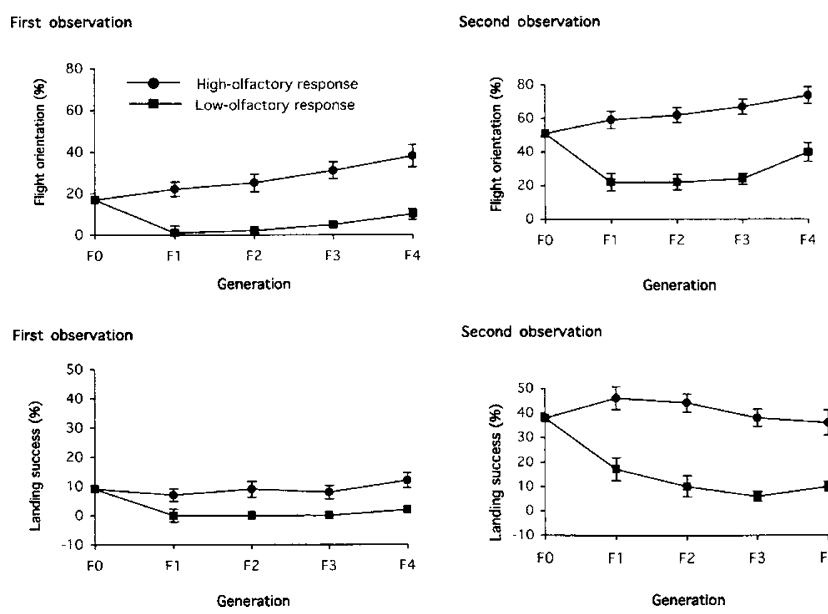

Second observation

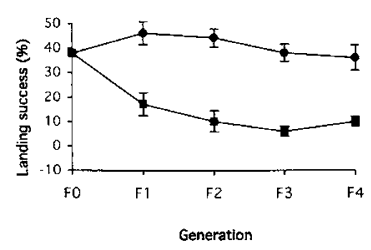

Figure 1 Changes in flight orientation and landing success of C. glomerata females due to selection for high- and low-olfactory response to hexane extract from herbivore-infested plants. The mean percentages and their standard errors are shown with respect to different generations of selection. Each individual was observed twice in a wind tunnel.

Table 1 Realized heritabilities $\left(h^{2}\right)$ for flight orientation and landing success in the response of female C. glomerata to hexane extract from a plant-host complex

\begin{tabular}{lccc}
\hline & Upward selection & Downward selection & Mean \\
\hline Flight orientation & 0.313 & 0.184 & 0.248 \\
Landing success & 0.083 & 0.350 & 0.216 \\
\hline
\end{tabular}


When averaged from the two directions of selection, the realized heritability was slightly greater for flight orientation than for landing success.

Comparison of flight response between two observations As compared with the first observation, the second observation recorded more individuals displaying flight orientation to the odour source. Flight orientation in the high-olfactory response strain increased between 34 and $37 \%$ relative to the corresponding first observation, and in the low-olfactory response strain, it increased between 21 and $34 \%$ in different generations. The significance of differences between the two observations was statistically confirmed for both upward $(\mathrm{F}=57.66$, $\mathrm{df}=1,20$, $P<0.001)$ and downward $(\mathrm{F}=65.99, \mathrm{df}=1,18, P<0.001)$ directions. Likewise, there were also significant differences in landing success between the two observations, with the level increasing between 22 and $39 \%$ in the higholfactory response strain $(\mathrm{F}=123.83, \mathrm{df}=1,20, P<0.001)$ and between 6 and $29 \%$ in the low-olfactory response strain $(\mathrm{F}=51.85, \mathrm{df}=1,18, P<0.001)$.

Differences in flight response between the two observations were also shown in the magnitude of variability within each generation. As measured by the coefficient of variation (CV), variability in both flight orientation and landing success was consistently reduced in the second observation, regardless of the direction of selection (Figure 2).

Flight responses to hexane extract versus solvent alone The percentage of individuals showing flight orientation in both high- and low-olfactory response strains varied with the odour sources used in the bioassay (Figure 3). When only solvent (hexane) was used as an odour source, most wasps from both strains immediately took off, but did not fly towards the odour source. Such flight failed to meet the criteria for flight orientation, that is, directional and/or zigzag flight towards the odour source. In the low-olfactory response strain, only $3 \%$ of the wasps showed flight orientation to the solvent, as
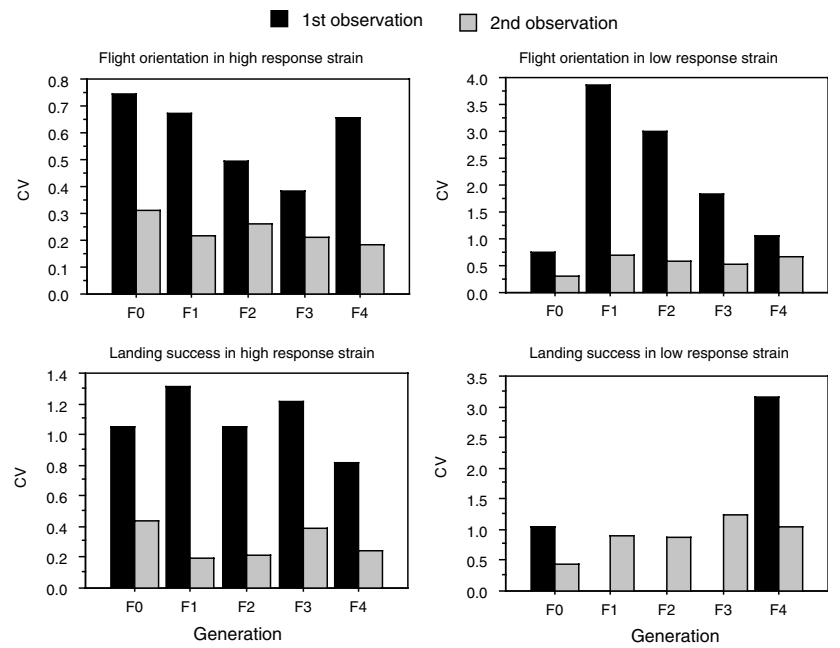

Figure 2 Comparisons of variability in the percentage of flight orientation and landing success between the first and second observations. The variability is presented by the $\mathrm{CV}$ within generations (note the different scales used).
Low-olfactory response strain

High-olfactory response strain

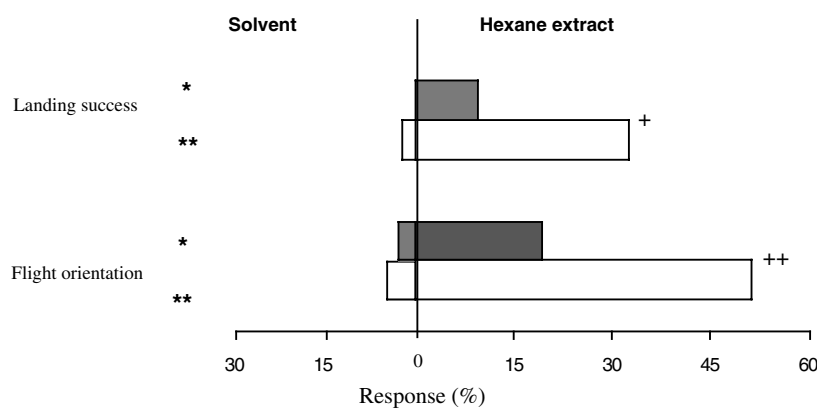

Figure 3 Behavioural responses or female C. glomerata exposed to either hexane extract or solvent hexane from herbivore-infested plants. Behavioural responses in the first observation were scored. * and $* *$ indicate significance at $P<0.05$ and 0.01 levels, respectively, based on logistic regression on odour sources within each of the high- and low-olfactory response strains, and ${ }^{+}$and ${ }^{++}$indicate significance at $P<0.05$ and 0.01 levels, respectively, based on logistic regression on strains.

compared to $19.4 \%$ when the hexane extract was used (logistic regression: $\chi^{2}=5.06, \mathrm{df}=1, P=0.024$ ). In the high-olfactory response strain, $4.8 \%$ of the wasps displayed flight orientation to the solvent alone, while $51.2 \%$ flew to the hexane extract (logistic regression: $\left.\chi^{2}=25.77, \mathrm{df}=1, P<0.01\right)$. Likewise, more wasps in both the strains landed successfully on the gauze in response to the hexane extract than to the solvent hexane alone (logistic regression: $\chi^{2}=4.70, \mathrm{df}=1, P<0.05$ for the lowolfactory response strain; $\chi^{2}=15.53, \mathrm{df}=1, P<0.01$ for the high-olfactory response strain).

When tested for response to the solvent alone, no significant differences were observed between the two strains either in flight orientation (logistic regression: $\left.\chi^{2}=0.56, \mathrm{df}=1, P>0.05\right)$ or in landing success $\left(\chi^{2}=1.58\right.$, $\mathrm{df}=1, P>0.05)$. In response to the hexane extract, however, the high-olfactory response strain showed a greater percentage than did the low-olfactory response strain in both flight orientation (logistic regression: $\left.\chi^{2}=7.41, \mathrm{df}=1, P<0.01\right)$ and landing success $\left(\chi^{2}=5.76\right.$, $\mathrm{df}=1, P<0.05)$. These results clearly demonstrated that the two selected strains were genetically differentiated in terms of olfactory perception rather than upwind flight ability.

\section{Discussion}

The population of C. glomerata significantly responded to bidirectional selection on the innate flight response to semiochemicals from a plant-host complex. In comparison to the base population, the upward selection increased the level of flight orientation in response to the odour source, while the downward selection achieved the opposite changes. Results from the control experiment excluded upwind flight ability as a factor responsible for differences in the innate flight response between the two strains, although the upwind flight of insects is a behaviour that can be changed by directional selection (Weber, 1996). The directional selection also led to significant differentiation in landing success between the two strains. Correlated responses in flight orientation 
and landing success indicate that they are two integrated components of the host-foraging behaviour of parasitoids.

As compared with the first observation, the level of flight response to the hexane extract significantly increased in the second observation. Such an increase in the olfactory response following the first exposure to the innately recognized stimuli might be the result of olfactory sensitization according to Papaj and Prokopy (1989). The increase of olfactory response in parasitoids is often shown by offering the test wasps direct contact with their hosts and/or host by-products (Tumlinson et al, 1993). In C. glomerata, for example, the flight response of female wasps to a plant-host complex significantly increased after walking on the host-infested cabbage leaves with host silk and faeces for $20 \mathrm{~s}$ or experiencing oviposition on the host (Steinberg et al, 1992). The results of the present study demonstrated that in C. glomerata, olfactory sensitization resulted from even a brief pre-exposure to semiochemicals from a plant-host complex, without direct contact with any host or host byproducts, leading not only to the reinforcement of responsiveness but also to a reduction in phenotypic variability, regardless of genetic differences. In the parasitoids like C. glomerata that have multiple, polyphagous hosts, such olfactory sensitization might have evolved in dealing with the complexity and variability of habitats (Vet et al, 1995).

Divergent changes between the two selected strains in the innate flight response due to directional selection provide direct evidence of genetic effect on the odourguided behaviour of C. glomerata. Although the lowolfactory response strain reached the bottom limit after the first generation of selection, the high-olfactory response strain showed no sign of reaching a selection limit, even after four generations of selection. The steady divergence of the two selected strains suggests that multiple genes affect the olfactory response of parasitoids. The realized heritability derived from the selection experiment further indicates the magnitude of such a genetic influence. The realized heritabilities estimated for both flight orientation and landing success in C. glomerata are less than 0.5 , as is seen for most behavioural characters in animals (Plomin, 1990), and also below the range $(0.44-0.82)$ of those estimated for the responsiveness of the predatory mite Phytoseiulus persimilis Athias-Henriot to herbivore-infested plants (Margolies et al, 1997). The results derived from the selection experiment suggest the presence of an additive genetic variance in the olfactory response of C. glomerata, even though most of the variability observed in the odour-guided behaviour might not have a genetic origin.

The experimental finding of additive genetic variance apparently contradicts our general expectation. A high positive response of foraging parasitoids to volatiles from herbivore-infested plants would contribute to a high reproductive success (Vet, 2001), and natural selection should favour the genotypes with a strong response to these semiochemicals and hence eliminate the additive genetic variance (Bulmer, 1976). The presence of genetic variation in olfactory response may be explained by the habitat heterogeneity of the parasitoid species concerned. C. glomerata is regarded as a generalist parasitoid, as in the field it exploits at least five Pieris species as hosts, and each of these hosts feeds on various cruciferous and noncruciferous plants (Laing and Levin, 1982). When infested by different herbivore species, a single plant can emit different volatile chemicals, since the plant volatiles are often herbivorespecific (De Moraes et al, 1998; Shiojiri et al, 2001). For C. glomerata, the availability of suitable host plants and/or herbivores varies with season and geographical location (Feltwell, 1982; Ohsaki and Sato, 1994), and the composition of volatile chemicals changes according to the combination of host plants and herbivores (Brodeur et al, 1996; Benrey et al, 1997). Thus, various resources of semiochemicals are available simultaneously or sequentially to a population of C. glomerata, so that different genotypes may be subject to quite strong selection for differential resource utilization, and, as a consequence, the genetic variation may persist in the natural population (Taylor, 1976). Indeed, a relationship between the genetic variability of olfactory responses and the diversity of volatile chemicals was shown in Drosophila melanogaster Meigen (Alcorta and Rubio, 1989). Although the hypothesis that natural selection in olfactory response acts through habitat selection (Hoffmann et al, 1984) remains to be tested for the case of C. glomerata, the presence of genotypes with differential olfactory responses in natural populations should have an adaptive value for the generalist parasitoid, to optimize host selection and utilization in the natural habitats.

\section{Acknowledgements}

We thank Letizia Mattiacci and Alan Hern for valuable advice on bioassay, and Henry Wöhrnschimmel, David Wettstein, Lena Obrist and Tanja Christoffel for assistance with insect culture. We are also very grateful to Kathrin Tschudi-Rein, Anja Rott and two anonymous reviewers for their helpful comments on the manuscript.

\section{References}

Alcorta E, Rubio J (1989). Intrapopulational variation of olfactory responses in Drosophila melanogaster. Behav Genet 19: 285-299.

Benrey B, Denno RF, Kaiser L (1997). The influence of plant species on attraction and host acceptance in Cotesia glomerata (Hymenoptera: Braconidae). J Insect Behav 10: 619-630.

Brodeur J, Geervliet JBF, Vet LEM (1996). The role of host species, age and defensive behaviour on ovipositional decisions in a solitary specialist and gregarious generalist parasitoid (Cotesia species). Entomol Exp Appl 81: 125-132.

Bulmer MG (1976). The effect of selection on genetic variability: a simulation study. Genet Res 28: 101-117.

Campan E, Couty A, Carton Y, Phamdelègue MH, Kaiser L (2002). Variability and genetic components of innate fruit odour recognition in a parasitoid of Drosophila. Physiol Entomol 27: 243-250.

De Moraes CM, Lewis WJ, Paré PW, Alborn HT, Tumlinson JH (1998). Herbivore-infested plants selectively attract parasitoids. Nature 393: $570-573$

Dicke M (1994). Local and systemic production of volatile herbivore-induced terpenoids: their role in plant-carnivore mutualism. J Plant Physiol 143: 465-472.

Falconer DS (1989). Introduction to Quantitative Genetics, (3rd Edn.) John Wiley and Sons: New York.

Feltwell J (1982). The Large White Butterfly: Biology, Biochemistry and Physiology of Pieris brassicae (Linnaeus), The Hague: London. 
$\mathrm{Gu} \mathrm{H}$, Dorn S (2000). Genetic variation in behavioral response to herbivore-infested plants in the parasitic wasp, Cotesia glomerata (L.) (Hymenoptera: Braconidae). J Insect Behav 13: 141-156.

$\mathrm{Gu} \mathrm{H}$, Dorn S (2001). How do wind velocity and light intensity influence host-location success in Cotesia glomerata (Hym., Braconidae)? J Appl Entomol 125: 115-120.

Hoffmann AA, Parsons PA, Nielsen KM (1984). Habitat selection: olfactory response of Drosophila melanogaster depends on resources. Heredity 53: 139-143.

Laing JE, Levin DB (1982). A review of the biology and a bibliography of Apanteles glomeratus (L.) (Hymenoptera: Braconidae). Bioc News Inf 3: 7-23.

Lewis WJ, Vet LEM, Tumlinson JH, van Lenteren JC, Papaj DR (1990). Variations in parasitoid foraging behavior: essential element of a sound biological control theory. Environ Entomol 19: 1183-1193.

Margolies DC, Sabelis MW, Boyer JE (1997). Response of a phytoseiid predator to herbivore-induced plant volatiles: selection on attraction and effect on prey exploitation. J Insect Behav 10: 695-709.

Mattiacci L, Dicke M, Posthumus MA (1995). $\beta$-Glucosidase: an elicitor of herbivore-induced plant odor that attracts host-searching parasitic wasps. Proc Natl Acad Sci USA 92: 2036-2040.

Mattiacci L, Rocca BA, Scascighini N, D'Alessandro M, Hern A, Dorn S (2001). Systemically induced plant volatiles emitted at the time of "danger". J Chem Ecol 27: 2233-2252.

Ohsaki N, Sato Y (1994). Food plant choice of Pieris butterflies as a trade-off between parasitoid avoidance and quality of plants. Ecology 75: 59-68.

Papaj DR, Prokopy RJ (1989). Ecological and evolutionary aspects of learning in phytophagous insects. Annu Rev Entomol 34: 315-350.
Plomin R (1990). The role of inheritance in behavior. Science 248 183-188.

Prevost G, Lewis WJ (1990). Heritable differences in the response of the braconid wasp Microplitis croceipes to volatile allelochemicals. J Insect Behav 3: 277-287.

Shiojiri K, Takabayashi J, Yano S (2001). Infochemically mediated tritrophic interaction webs on cabbage plants. Popul Ecol 43: 23-29.

Steinberg S, Dicke M, Vet LEM, Wanningen R (1992). Response of the braconid parasitoid Cotesia (=Apanteles) glomerata to volatile infochemicals: effects of bioassay set-up, parasitoid age and experience and barometric flux. Entomol Exp Appl 63: 163-175.

Taylor CE (1976). Genetic variation in heterogeneous environments. Genetics 83: 887-894.

Tumlinson JH, Turlings TCJ, Lewis WJ (1993). Semiochemically mediated foraging behavior in beneficial parasitic insects. Arch Insect Biochem Physiol 22: 385-391.

Turlings TCJ, Tumlinson JH, Lewis WJ (1990). Exploitation of herbivore-induced plant odors by host-seeking parasitic wasps. Science 250: 1251-1253.

Vet LEM (2001). Parasitoid searching efficiency links behaviour to population processes. Appl Entomol Zool 36: 399-408.

Vet LEM, Dicke M (1992). Ecology of infochemical use by natural enemies in a tritrophic context. Annu Rev Entomol 67: 141-172.

Vet LEM, Lewis WJ, Cardé RT (1995). Parasitoid foraging and learning. In: Bell W, Cardé (eds), Chemical Ecology of Insects, Chapman \& Hall: London. pp 65-101.

Vinson SB (1976). Host selection by insect parasitoids. Annu Rev Entomol 21: 109-133.

Weber KE (1996). Large genetic change at small fitness cost in large populations of Drosophila melanogaster selected for wind tunnel flight: rethinking fitness surfaces. Genetics 144: 205-213. 